\title{
On the Geometry of Hamiltonian Symmetries
}

\author{
Narmanov Abdigappar*, Parmonov Hamid \\ Faculty of Mathematics, National University of Uzbekistan, Tashkent, 100174, Tashkent, Uzbekistan
}

Received February 10, 2020; Revised March 2, 2020; Accepted March 12, 2020

Copyright $@ 2020$ by authors, all rights reserved. Authors agree that this article remains permanently open access under the terms of the Creative Commons Attribution License 4.0 International License

\begin{abstract}
The problem of integrating equations of mechanics is the most important task of mathematics and mechanics. Before Poincare's book "Curves Defined by Differential Equations", integration tasks were considered as analytical problems of finding formulas for solutions of the equation of motion. After the appearance of this book, it became clear that the integration problems are related to the behavior of the trajectories as a whole. This, of course, stimulated methods of qualitative theory of differential equations.
\end{abstract}

Present time, the main method in this problem has become the symmetry method. Newton used the ideas of symmetry for the problem of central motion. Further, Lagrange revealed that the classical integrals of the problem of gravitation bodies are associated with invariant equations of motion with respect to the Galileo group. Emmy Noether showed that each integral of the equation of motion corresponds to a group of transformations preserving the action. The phase flow of the Hamilton system of equations, in which the first integral serves as the Hamiltonian, translates the solutions of the original equations into solutions. The Liouville theorem on the integrability of Hamilton equations was created on this idea. The Liouville theorem states that phase flows of involutive integrals generate an Abelian group of symmetries

Hamiltonian methods have become increasingly important in the study of the equations of continuum mechanics, including fluids, plasmas and elastic media.

In this paper it is considered the problem on the Hamiltonian system which describes of motion of a particle which is attracted to a fixed point with a force varying as the inverse cube of the distance from the point. We are concerned with just one aspect of this problem, namely the questions on the symmetry groups and Hamiltonian symmetries. It is found Hamiltonian symmetries of this Hamiltonian system and it is proven that Hamiltonian symmetry group of the considered problem contains two dimensional Abelian Lie group. Also it is constructed the singular foliation which is generated by infinitesimal symmetries which invariant under phase flow of the system.

In the present paper, smoothness is understood as smoothness of the class $C^{\infty}$.

Keywords Poisson Bracket, Poisson Manifold, Hamilto- nian Vector Field, Hamiltonian System, Symmetry Group

\section{Introduction}

Given a smooth manifold $M$ a Poisson bracket on $M$ assigns to each pair of smooth, real-valued functions $F, H: M \rightarrow R$ another smooth, real-valued function, which we denote by $F, H$. There are certain basic properties that such a bracket operation must satisfy in order to qualify as a Poisson bracket.

Definition -1. A Poisson bracket on a smooth manifold $M$ is an operation that assigns a smooth real-valued function $\{F, H\}$ on $M$ to each pair $F, H$ of smooth, real-valued functions, with the basic properties:

(1) Bilinearity: $\{\lambda F+\mu P, H\}=\lambda\{F, H\}+\mu\{P, H\}$,

$\{F, \lambda H+\mu P\}=\lambda\{F, H\}+\mu\{F, P\}$ for constants $\lambda, \mu \in$ $R$,

(2) Skew-Symmetry: $\{F, H\}=-\{H, F\}$,

(3) Jacobi Identity: $\{\{F, H\}, P\}+\{\{P, F\}, H\}+$ $\{\{H, P\}, F\}=0$

(4) Leibniz' Rule: $\{F, H \cdot P\}=\{F, H\} \cdot P+H \cdot\{F, P\}$.

(Here $\cdot$ denotes the ordinary multiplication of real-valued functions.)

A manifold $M$ with a Poisson bracket is called a Poisson manifold, the bracket defining a Poisson structure on $M$. The notion of a Poisson manifold is slightly more general than that of a symplectic manifold, or manifold with Hamiltonian structure; in particular, the underlying manifold $M$ need not be even-dimensional. This is borne out by the standard examples from classical mechanics.

Let $M$ be the Euclidean space $R^{m}$ with coordinates

$$
(p, q, z)=\left(p^{1}, p^{2} \ldots, p^{n}, q^{1}, q^{2} \ldots, q^{n}, z^{1}, z^{2} \ldots, z^{l}\right),
$$

where $2 n+l=m$. If $F(p, q, z), H(p, q, z)$ are smooth functions, we define their Poisson bracket to be the function

$$
\{F, H\}=\sum_{i=1}^{n} \frac{\partial F}{\partial q^{i}} \frac{\partial H}{\partial p^{i}}-\sum_{i=1}^{n} \frac{\partial F}{\partial p^{i}} \frac{\partial H}{\partial q^{i}} .
$$


This bracket is clearly bilinear and skew-symmetric; the verifications of the Jacobi identity and the Leibniz rule are simple exercises.

We note the particular bracket identities

$$
\left\{p^{i}, p^{j}\right\}=0,\left\{q^{i}, q^{j}\right\}=0,\left\{q^{i}, q^{j}\right\}=\delta_{i}^{j},
$$

in which $i$ and $j$ run from 1 to $\mathrm{n}$, and $\delta_{i}^{j}$ is the Kronecker symbol.

Definition-2. Let $M$ be a Poisson manifold. A smooth, real-valued function $C: M \rightarrow R$ is called a distinguished function if the Poisson bracket of $C$ with any other real-valued function vanishes identically, i.e. $\{C, H\}=0$ for all $H: M \rightarrow$ $R$.

In the case of the canonical Poisson bracket on $R^{m}$ the only distinguished functions are the constants, which always satisfy the requirements of the definition.

Definition -3. Let $M$ be a Poisson manifold and $H: M \rightarrow$ $R$ a smooth function. The Hamiltonian vector field associated with $\mathrm{H}$ is the unique smooth vector field $X_{H}$ on $M$ satisfying $X_{H}(F)=\{F, H\}=-\{H, F\}$ for every smooth function $F: M \rightarrow R$.

In the case of the canonical Poisson bracket on $R^{m}, m=$ $2 n+l$, the Hamiltonian vector field corresponding to $H(p, q, z)$ is clearly

$$
X_{H}=\sum_{i=1}^{n}\left\{\frac{\partial H}{\partial p^{i}} \frac{\partial}{\partial q^{i}}-\frac{\partial H}{\partial q^{i}} \frac{\partial}{\partial p^{i}}\right\} .
$$

The corresponding flow is obtained by integrating the system of ordinary differential equations

$$
\frac{d p^{i}}{d t}=-\frac{\partial H}{d q^{i}}, \frac{d q^{i}}{d t}=\frac{\partial H}{d p^{i}}, \frac{d z^{l}}{d t}=0 .
$$

There is a fundamental connection between the Poisson bracket of two functions and the Lie bracket of their associated Hamiltonian vector fields, which forms the basis of much of the theory of Hamiltonian systems. It is well known following theorem [6].

Theorem-1. Let $M$ be a Poisson manifold and let $F, H$ : $M \rightarrow R$ be smooth functions with corresponding Hamiltonian vector fields $X_{F}, X_{H}$. The Hamiltonian vector field associated with the Poisson bracket of $\mathrm{F}$ and $\mathrm{H}$ is the Lie bracket of the two Hamiltonian vector fields:

$$
X_{\{F, H\}}=-\left[X_{F}, X_{H}\right] .
$$

The Hamiltonian vector field associated with $H(x)$ has the form

$$
X_{H}=\sum_{i=1}^{m}\left\{\sum_{j=1}^{m} J^{i j}(x) \frac{\partial H}{\partial x^{j}}\right\} \frac{\partial}{\partial x^{i}},
$$

where $J^{i j}(x)=\left\{x^{i}, x^{j}\right\}$.

\section{MAIN PART}

Let us consider system of ordinary differential equations in Hamilton's form

$$
\frac{d x}{d t}=J(x) \nabla H(x)
$$

where $H(x)$ - Hamiltonian, $J^{i j}(x)=\left\{x^{i}, x^{j}\right\}$ - the structure matrix , defining Poisson bracket.

Example-1. On the plane, the Hamiltonian vector field corresponding to the function

$$
H(p, q)=\frac{1}{2}\left(p^{2}+q^{2}\right)
$$

has the form.

$$
X_{H}=-q \frac{\partial}{\partial p}+p \frac{\partial}{\partial q} .
$$

The corresponding Hamiltonian system of differential equations

$$
\frac{d p}{d t}=-q, \frac{d q}{d t}=p
$$

Definition -4. A function $P(x, t)$ is called the first integral of system (4) if for the solution $x(t)$ it holds $P(x(t), t)=$ const for all $t$.

For system (5), the function

$$
H(p, q)=\frac{1}{2}\left(p^{2}+q^{2}\right)
$$

is the first integral.

The first integrals are easily described using the Poisson bracket. The following theorem is known ([6], p. 494) on the first integrals of the system

Theorem-2. The function $P(x, t)$ is the first integral of the Hamiltonian system (4) if and only if

$$
\frac{d P}{d t}+\{P, H\}=0 .
$$

for all $t$. In particular, a time-independent function $P(x)$ is the first integral if and only if

$$
\{P, H\}=0 .
$$

everywhere.

The following corollary follows from this theorem.

Corollary 1. If the Hamiltonian $H$ of the Hamiltonian system (4) does not depend on time, then it itself is automatically the first integral.

In fact, the function

$$
H(p, q)=\frac{1}{2}\left(p^{2}+q^{2}\right)
$$

is also the first integral of system (5).

Corollary 2. Any distinguished function with respect to the Poisson bracket given by the matrix $J^{i j}(x)=\left\{x^{i}, x^{j}\right\}$ is the first integral of the Hamiltonian system (4).

Let us recall that one parametrical group of transformations is called symmetry group of system (4), each element of the group transforms solutions of system (4) to other solutions of (4.)Symmetry group of system (4) is called Hamiltonian symmetry group if its infinitesimal generator is Hamiltonian vector field. In this case infinitesimal generator of symmetry group is called infinitesimal symmetry (4).

For systems of Euler-Lagrange equations, first integrals arise from variational symmetry groups; for Hamiltonian systems 
this role is played by the one-parameter Hamiltonian symmetry groups.

Example-2. The Hamiltonian vector field

$$
W=-\frac{p}{p^{2}+q^{2}} \frac{\partial}{\partial p}-\frac{q}{p^{2}+q^{2}} \frac{\partial}{\partial q}
$$

corresponding to the function

$$
P(p, q)=\operatorname{arctang} \frac{q}{p}
$$

is Hamiltonian symmetry for the system (5).

Flow of this vector field consists following diffeomorphisms:

$$
X^{s}:(p, q) \rightarrow\left\{\begin{array}{l}
p(s)=\frac{p}{\rho} \sqrt{\rho^{2}-2 s} \\
q(s)=\frac{q}{\rho} \sqrt{\rho^{2}-2 s}
\end{array}\right.
$$

where $\rho=\sqrt{p^{2}+q^{2}}$.

Every diffeomorphism $X^{s}$ translates trajectory $(p(t), q(t))$ of the system (5) to following curves

$$
\left\{\begin{array}{l}
p^{s}(t)=\frac{p(t)}{\rho_{0}} \sqrt{\rho_{0}^{2}-2 s} \\
q^{s}(t)=\frac{q(t)}{\rho_{0}} \sqrt{\rho_{0}^{2}-2 s}
\end{array}\right.
$$

We can check that

$$
\left\{\begin{array}{l}
\frac{d}{d t} p^{s}(t)=-q^{s}(t) \\
\frac{d}{d t} q^{s}(t)=p^{s}(t)
\end{array} .\right.
$$

It follows from here for every $s$ a curve $\left(p^{s}(t), q^{s}(t)\right)$ is a trajectory of the system (5). Thus vector field (8) is a Hamiltonian symmetry for the system (5).

This example shows that if vector field $X_{P}$ is infinitesimal symmetry of (4), the function $P(x, t)$ is not necessarily first integral for the system (4).

In this case it holds following theorem.

Theorem 3. Let system (4) does not dependent from $t, X_{P}$ is a Hamiltonian symmetry of (4), where the function $P$ - does not dependent from $t$. Then, for each solution $x(t)$ of system (4), it holds $P(x(t), t)=a t+b$ for all $t$, where $a, b-$ are constant.

Proof. If the function $P(x, t)$ is the first integral, then it is obvious that for each solution $x(t)$ of system (1) it holds $P(x(t), t)=b$ for all $t$.

Let $X_{P}$ be a Hamiltonian symmetry of (4).The symmetry condition implies that the Hamiltonian vector field associated with the function $d P / d t+\{P, H\}$ vanishes everywhere, and hence this combination must be a distinguished function $C(x, t): d P / d t+\{P, H\}=C(x, t)$.

Since the function $H, P$ are independent of, we have $\{P, H\}=C=a$ is a constant function.

We put $G=\int_{0}^{t}\{P, H\} d t=a t+b$. Along the solution of system (1) takes place $d G / d t=a$.
Now we can verify that the function $P_{0}=P-G$ is the first integral: $d P_{0} / d t+\left\{P_{0}, H\right\}=-d G / d t+\{P, H\}=0$.

Thus, for each solution to system (4) it holds $P(x(t), t)=$ $a t+b$ for all $t$, where $a, b-$ are constant. Proof is complete.

Let us consider Hamiltonian system

$$
\frac{d p_{1}}{d t}=0, \frac{d p_{2}}{d t}=\frac{p_{1}^{2}-1}{q_{2}^{3}}, \frac{d q_{1}}{d t}=\frac{p_{1}}{q_{2}^{2}}, \frac{d q_{2}}{d t}=p_{2}
$$

where $H\left(p_{1}, p_{2}, q_{1}, q_{2}\right)=\frac{1}{2}\left\{p_{2}^{2}+\frac{p_{1}^{2}}{q_{2}^{2}}-\frac{1}{q_{2}^{2}}\right\}$.

This system is a system of equations of motion of a particle which is attracted to a fixed point with a force varying as the inverse cube of the distance: $q_{2}$ and $q_{1}$ are respectively the radius vector and vectorial angle of the particle referred to the center of force.

In [9] by integrating this system it is found the equation of the orbit described by the particle in polar coordinates. We will find Hamiltonian symmetries of system (9)which allow us to find first integrals.

Theorem 4. The Hamiltonian vector fields associated with functions

$$
P_{1}=\frac{1}{2} p_{1}^{2}, P_{2}=\frac{1}{2} \frac{p_{2}^{2}}{p_{1}^{2}-1}-\frac{1}{2} \frac{1}{q_{2}^{2}} .
$$

are Hamiltonian symmetries of Hamiltonian system (9).

Proof. Let us to find Hamiltonian vector fields associated with functions $P_{1}, P_{2}, P_{3}$.

$$
\begin{gathered}
X_{P_{1}}=p_{1} \frac{\partial}{\partial q_{1}}, \\
X_{P_{2}}=-\frac{1}{q_{2}^{3}} \frac{\partial}{\partial p_{2}}-\frac{p_{1} p_{2}^{2}}{\left(p_{1}^{2}-1\right)^{2}} \frac{\partial}{\partial q_{1}}+\frac{p_{2}}{p_{1}^{2}-1} \frac{\partial}{\partial q_{2}},
\end{gathered}
$$

We need also to find Hamiltonian vector field associated with functions $H$.

$$
X_{H}=\frac{p_{1}^{2}-1}{q_{2}^{3}} \frac{\partial}{\partial p_{2}}+\frac{p_{1}}{q_{2}^{3}} \frac{\partial}{\partial q_{1}}+p_{2} \frac{\partial}{\partial q_{2}} .
$$

By the Theorem-1 we have

$$
\left[X_{P_{i}}, X_{H}\right]=-X_{\left\{P_{i}, H\right\}}
$$

for every function $P_{i}$.

Now by using formula (1) we will calculate Poisson brackets of the of functions $H$ and $P_{i}:\left\{P_{i}, H\right\}=0$.

It follows from here

$$
\left[X_{P_{i}}, X_{H}\right]=0
$$

It is well known in this case the flow of vector field $X_{P_{i}}$ maps solutions of system (9) into solutions, i.e. one parametric group of transformations generated by vector field $X_{P_{i}}$ is Hamiltonian symmetry group of the system (9). Proof of the theorem is complete.

It is easy to check for the Lie brackets $\left[X_{P_{1}}, X_{P_{2}}\right]$ of vector fields $X_{P_{1}}, X_{P_{2}}$ it holds equality:

$$
\left[X_{P_{1}}, X_{P_{2}}\right]=0 .
$$


It follows from this equality

Corollary 3. Hamiltonian symmetry group of the system (9) contains two dimensional Lie group whose Lie algebra is generated by vector fields $X_{P_{1}}, X_{P_{2}}$.

Now we will study of geometry of orbits of vector fields $X_{P_{1}}, X_{P_{2}}$.

Let us recall a notion of orbit. Let $D$ be a system of smooth vector fields defined on $M$. The system $D$ can contain finitely or infinitely many smooth vector fields. For a vector field $X \in$ $D$, by $X^{t}(x)$ we denote the integral curve of $X$ passing through a point $x \in M$ for $t=0$. The mapping $t \longrightarrow X^{t}(x)$ is defined in some domain $I(x)$, which in general depends not only on the field $X$ but also on the initial point $x$.

Throughout the following, we assume that $t \in I(x)$. If the domain $I(x)$ of the curve $t \longrightarrow X^{t}(x)$ coincides with the real line for each $x \in M$, then the vector field $X$ is said to be complete.

Definition-5. The orbit $L(x)$ of a family $D$ of vector fields through a point $x$ is the set of points $y$ in $M$ such that there exist real numbers $t_{1}, t_{2}, \ldots, t_{k}$ and vector felds $X_{i_{1}}, X_{i_{2}}, \ldots, X_{i_{k}}$ in $D$ (where $k$ is an arbitrary positive integer) such that

$$
y=X_{i_{k}}^{t_{k}}\left(X_{i_{k-1}}^{t_{k-1}}\left(\ldots\left(X_{i_{1}}^{t_{1}}\right) \ldots\right)\right) .
$$

Obviously, if the family $D$ consists of a single vector field, then the orbit is a smooth curve (a one-dimensional manifold).

Numerous investigations have been devoted to the study of geometry of orbits of vector fields [1],[4]-[8].

The fundamental result in study of orbits is Sussmann theorem [8], which asserts that every orbit of smooth vector fields with Sussmann topology has differential structure with respect to which it is a immersed submanifold of $M$.

Recall that a mapping $P$ that takes each point $x \in M$ to some subspace $P(x) \subset T_{x} M$ is called a distribution. If $\operatorname{dim}$ $P(x)=k$ for all $x \in M$, then $P$ is called a $k$-dimensional distribution. A distribution $P$ is said to be smooth if, for each point $x \in M$, there exists a neighborhood $U(x)$ of that point and smooth vector fields $X_{1}, X_{2}, \ldots, X_{m}$ defined on $U(x)$ such that the vectors

$$
X_{1}(y), X_{2}(y), \ldots, X_{m}(y)
$$

form a basis of the subspace $P(y)$ for each $y \in U(x)$. A family $D$ of smooth vector fields naturally generates the smooth distribution that takes each point $x \in M$ to the subspace $P(x)$ of the tangent space $T_{x} M$ spanned by the set

$$
D(x)=\{X(x): X \in D\} .
$$

Obviously, the dimension of the subspace $P(x)$ can vary from point to point.

A distribution $P$ is said to be completely integrable if, for each point $x \in M$, there exists a connected submanifold $N_{x}$ of the manifold $M$ such that $T_{y} N_{x}=P(y)$ for all $y \in N_{x}$.

The submanifold $N_{x}$ is called an integral submanifold of the distribution $P$. For a vector field $X$, we write $X \in P$ if $X(x) \in P(x)$ for all $x \in M$.

A distribution $P$ is said to be involutive if the inclusion $X, Y \in P$ implies that $[X, Y] \in P$, where $[X, Y]$ is the Lie bracket of the fields $X$ and $Y$.
The Frobenius theorem ([3], page 10) provides a necessary and suffcient condition for the completely integrability of a distribution of constant dimension.

Theorem Frobenius. A distribution $P$ on a manifold $M$ is completely integrable if and only if it is involutive.

Let $A(D)$ be the smallest Lie algebra containing the set $D$. By setting $A_{x}(D)=\{X(x): X \in A(D)\}$, we obtain an involutive distribution $P_{D}: x \rightarrow A x(D)$. If the dimension $\operatorname{dim} A_{x}(D)$ is independent of $x$, then the distribution $P_{D}: x \rightarrow$ $A x(D)$ is completely integrable by the Frobenius theorem.

If the dimension $\operatorname{dim} A_{x}(D)$ depends on $x$, then, as examples show, the distribution $P_{D}: x \rightarrow A_{x}(D)$ is not necessarily completely integrable.

The Frobenius theorem generalized by Hermann to distributions of variable dimension provides a necessary and sufficient condition for the complete integrability of distributions which is finitely generated [4].

Definition-6. A system of vector fields

$$
D=\left\{X_{1}, X_{2}, \ldots, X_{k}\right\}
$$

on $\mathrm{M}$ is in involution if there exist smooth real-valued functions $f_{i j}^{l}(x), x \in M, i, j, l=1, \ldots, k$ such that for each $(i, j)$ it takes

$$
\left[X_{i}, X_{j}\right]=\sum_{l=1}^{k} f_{i j}^{l}(x) X_{l} .
$$

Theorem(Herman). The system

$$
D=\left\{X_{1}, X_{2}, \ldots, X_{k}\right\}
$$

of smooth vector fields on $M$ generates completely integrable distribution if and only if it is in involution.

Theorem-5. The orbits of the system of Hamiltonian vector fields $D=\left\{X_{P_{1}}, X_{P_{2}}\right\}$ generate singular foliation, any leaf of which is invariant with respect flow of the system (9).

Proof. It follows the involutiveness of the system $D$ of Hamiltonian vector fields from equality (10). By Hermann theorem the system $D$ is completely integrable.

Since $X_{P_{i}}\left(P_{j}\right)=0$ for $i, j=1,2$, every orbit of the system $D$ is contained in the one of connected components of level surfaces

$$
N^{C}: P_{1}\left(p_{1}, p_{2}, q_{1}, q_{2}\right)=C_{1}, P_{2}\left(p_{1}, p_{2}, q_{1}, q_{2}\right)=C_{2},
$$

where $C=\left(C_{1}, C_{2}\right) \in R^{2}$.

As $T_{x} N^{C}=A_{x}(D)$ for every point $x \in N^{C}$ every connected component of level surface $N$ is integral submanifold for the distribution

$$
P_{D}: x \rightarrow A_{x}(D)
$$

where $T_{x} N-$ tangent space of the submanifold $N$ at the point $x \in N$.

Let $N-$ be one of connected components of level surface $N_{C}$. Then for every $x \in N$ we have $L(x) \subset N$, where $L(x)-$ a orbit of the system $D$. It follows from [4], [8] it holds $\operatorname{dim} L(x) \geq \operatorname{dim} A_{x}(D)$ for all $x$. By using this fact it is not difficult to check that $\operatorname{dim} L(x)=\operatorname{dim} N$ for every $x \in N$, i.e. 
$L(x), N$ are manifolds the same dimension. Therefore, the set $L(x)$ is an open subset of $N$.

Different orbits do not intersect by definition of a orbit. Since the the submanifold $N$ is a connected set, it cannot be a union of different orbits. It follows from here $L(x)=N$ for every $x \in N$.

Thus the orbits of the the system of Hamiltonian vector fields $D=\left\{X_{P_{1}}, X_{P_{2}}\right\}$ are connected components of level surfaces $N_{C}$, which generate singular foliation on $R^{4}$

It is also clear any leaf of this foliation is invariant under flow of the system (9). Proof of the theorem-5 is complete.

Let us consider functions

$$
H(p, q)=\frac{1}{2} p_{2}^{2}+\frac{1}{2} \frac{p_{1}^{2}}{q_{2}^{2}}-\frac{1}{2} \frac{1}{q_{2}^{2}}, P_{2}=\frac{1}{2} \frac{p_{2}^{2}}{p_{1}^{2}-1}-\frac{1}{2} \frac{1}{q_{2}^{2}} .
$$

This functions satisfy conditions Liouville-Arnold theorem.

In dynamical systems theory, the Liouville -Arnold theorem states that if, in a Hamiltonian dynamical system with $n$ degrees of freedom, there are also known $n$ first integrals of motion that are independent and in involution, then there exists a canonical transformation to action-angle coordinates in which the transformed Hamiltonian is dependent only upon the action coordinates and the angle coordinates evolve linearly in time. Thus the equations of motion for the system can be solved in quadratures if the canonical transform is explicitly known [2].

Using Liouville- Arnold theorem we can formulate following theorem.

Theorem 6. 1.Functions (15) are in involution i.e $\left\{P_{2}, H\right\}=0$

2. The level manifold

$$
N^{C}: H\left(p_{1}, p_{2}, q_{1}, q_{2}\right)=C_{1}, P_{2}\left(p_{1}, p_{2}, q_{1}, q_{2}\right)=C_{2},
$$

where $C=\left(C_{1}, C_{2}\right) \in R^{2}$, is invariant with respect to the phase flow of the system (9);

3. The orbits of the system of Hamiltonian vector fields $D=$ $\left\{X_{H}, X_{P_{2}}\right\}$ generate singular foliation, any leaf of which is invariant with respect flow of the system (9).

Proof. Statement (1) is clear: $\left\{P_{2}, H\right\}=0$; Statement (2) of the Theorem- 6 follows from the Liouville- Arnold theorem. The proof of Statement (3) is similar to the proof of the theorem-5.

Comments. I. We can consider differentiable map

$$
\varphi: R^{2} \rightarrow N
$$

where

$$
\varphi(t, s)=X^{t}\left(Y^{s}(p, q)\right),
$$

$X^{t}, Y^{s}$ are respectively flows of vector fields $X_{H}$ and $X_{P_{2}}$, $(p, q) \in N, N$ - connected component of level surface.

At regular points rank of $\varphi$ equal 2, at singular points it is 1 . Rank of $\varphi$ equal to the rank of vectors $X_{H}$ and $X_{P_{2}}$.

If the point $(p, q)-$ is regular, the leaf (orbit) is diffeomorphic to $R^{2}$.

At points $p_{1}=0, p_{2}=0$ vector fields $X_{H}$ and $X_{P_{2}}$ are collinear, that's why at this points orbits of singular foliation is a one dimensional manifold. This one dimensional manifold is given by equations

$$
p_{1}=0, q_{1}=0, q_{2}=\text { const. }
$$

At points $p_{1}=0, p_{2}=1$ vector fields $X_{H}$ and $X_{P_{2}}$ are not collinear. It follows from here rank of $\varphi$ equal 2 , therefore at this point orbits of singular foliation is a two dimensional manifold. This two dimensional manifold is given by equations

$$
p_{1}=0, q_{1}=\text { const. }
$$

II. If we consider Hamiltonian system

$\frac{d p_{1}}{d t}=0, \frac{d p_{2}}{d t}=-\frac{1}{q_{2}^{3}}, \frac{d q_{1}}{d t}=-\frac{p_{1} p_{2}^{2}}{\left(p_{1}^{2}-1\right)^{2}}, \frac{d q_{2}}{d t}=\frac{p_{2}}{p_{1}^{2}-1}$,

associated with function

$$
P_{2}=\frac{1}{2} \frac{p_{2}^{2}}{p_{1}^{2}-1}-\frac{1}{2} \frac{1}{q_{2}^{2}},
$$

any leaf of foliation from theorem- 6 also is invariant with respect flow of this system.

\section{Acknowledgements}

We are very grateful to experts for their appropriate and constructive suggestions to improve this template.

\section{REFERENCES}

[1] Azamov A.A. and Narmanov A.Ya. On the Limit Sets of Orbits of Systems of Vector Fields, Differential Equations, 2004, vol. 40, no. 2, pp. 257-260.

[2] Borisov A.V., Mamaev I.S. Rigid Body Dynamics. Izhevsk: NIC Regular and Chaotic Dynamics, 2001.

$384 \mathrm{p}$.

[3] Kobayashi, Sh. and Nomizu, K., Foundations of Differential Geometry , New York, London: Interscience,1963, vol.1,329 pages

[4] Narmanov, A.Ya and Saitova, S.S., On the geometry of orbits of Killing vector fields, Differential Equations, 2014, vol. 50, no. 12 , pp. 1584-1591.

[5] Narmanov, A. Ya and Saitova, S.S., On the geometry of the reachability set of vector fields, Differential Equations, 53, pages 311-316 (2017).

[6] Olver, P.J., Applications of Lie Groups to Differential Equations.Springer, 1993.

[7] Olver, P.J., Differential invariants and invariant differential equations, Lie Groups and their Appl. 1 (1994), 177-192. 
[8] Sussman H, Orbits of families of vector fields and integrability of distributions. Transactions of the AMS, v. 180, June 1973. P. 171-188.

[9] Whittaker E.T. A Treatise on the analytical dynamics of particles and rigid bodies; With an introduction to the problem of three bodies. Cambridge: At the University Press,1917. 